This is the accepted version of the article: Leconte N., Ortmann F., Cresti, A., Roche S. Unconventional features in the quantum Hall regime of disordered graphene: percolating impurity states and Hall conductance quantization. Physical Review B, 93(11):2016, article 115404

Available at: https://doi.org/10.1103/PhysRevB.93.115404

All rights reserved 


\title{
Unconventional Features in the Quantum Hall Regime of Disordered Graphene : Percolating Impurity States and Hall Conductance Quantization
}

\author{
Nicolas Leconte, ${ }^{1}$ Frank Ortmann, ${ }^{2,3}$ Alessandro Cresti, ${ }^{4}$ and Stephan Roche ${ }^{1,5}$ \\ ${ }^{1}$ ICN2 - Institut Catala de Nanociencia i Nanotecnologia, \\ Campus UAB, 08193 Bellaterra (Barcelona), Spain* \\ ${ }^{2}$ Institute for Materials Science and Max Bergmann Center of Biomaterials, TU Dresden, 01062 Dresden, Germany \\ ${ }^{3}$ Dresden Center for Computational Materials Science (DCCMS), TU Dresden, 01062 Dresden, Germany \\ ${ }^{4}$ IMEP-LAHC (UMR CNRS/INPG/UJF 5130), Grenoble INP, \\ Minatec, 3, Parvis Louis Néel, BP 257, F-38016, Grenoble, France \\ ${ }^{5}$ ICREA - Institucio Catalana de Recerca $i$ Estudis Avançats, 08010 Barcelona, Spain
}

(Dated: November 15, 2014)

\begin{abstract}
We report on the formation of critical states in disordered graphene, at the origin of variable and unconventional transport properties in the quantum Hall regime, such as a zero-energy Hall conductance plateau in the absence of energy bandgap and Landau level degeneracy breaking. By using an efficient real-space transport methodology, we compute both the dissipative and Hall conductivities of large size graphene models with random distributions of single or double vacancies. By analyzing the scaling of transport coefficients with defect density, system size and magnetic length, we elucidate the origin of anomalous quantum Hall features coming from magnetic-field dependent impurity states that percolate at unconventional critical energies. These findings shed light on unidentified states and quantum transport anomalies reported experimentally.

PACS numbers: 72.80.Vp, 73.63.-b, 73.22.Pr, 72.15.Lh, 61.48.Gh
\end{abstract}

Introduction.- The role of disorder in the Quantum Hall Effect (QHE) [1] has been essentially related to the existence of a localization/delocalization transition between electronic states, with the formation of critical (extended) states at the center of Landau Levels (LL) [2]. In very clean samples, the presence of this transition is assured by the sample edges which force the formation of extended states, while bulk states are localized by the magnetic field. The robustness of the QHE in the bulk limit is guaranteed by the contribution of either weak impurity potentials satisfying the so-called weakness condition $[3,4]$, strong scattering centers sufficiently far away from each other [5-7], or smooth potentials with longrange spatial variation [7-15]. Whenever disorder becomes too strong, all QHE features eventually vanish away. The discovery of the half-integer QHE in clean graphene has become one of the fundamental properties driven by the new quantum degree of freedom (i.e. pseudospin) and the related Berry's phase [16-18]. The electronic properties of graphene for large enough magnetic fields are characterized by the presence of a four-fold degenerate zero energy LL (where electrons and holes coexist) together with non-equidistant LLs at energies $E_{n}=\operatorname{sgn}(n) \sqrt{2 \hbar v_{F}^{2} e B|n|}\left(v_{F}=10^{6} \mathrm{~ms}^{-1}\right.$ is the Fermi velocity, $B$ is the magnetic field and $n$ is the integer LL index) $[19,20]$. This electronic spectrum results in a Hall conductance quantization $\sigma_{\mathrm{xy}}=\frac{4 e^{2}}{h}\left(n+\frac{1}{2}\right)$ [18], which is typically weakly affected by electron-hole puddles or weak surface disorder, but can exhibit further fragmentation of the plateaus structure whenever additional symmetry-breaking mechanisms lift the four-fold degeneracy of LLs $[21,22]$. The presence of an addi- tional quantized Hall plateau $\sigma_{x y}=0$ at low energy in high-mobility samples has been for instance assigned to Zeeman interaction (spin degeneracy lifting) or the formation of quantum Hall ferromagnetism [23-25]. The scaling of the zero-energy split gap with magnetic field remains however under scrutiny, with a behavior changing from linear to $\sqrt{B}$-like, depending on the underlying dominant symmetry breaking or on the sample quality $[25,26]$.

Recently, several experiments have reported unidentified electronic features in the quantum Hall regime, tentatively related to impurity states [27-30]. Additionally, the observation of a quantized Hall conductance in highly resistive (millimeter-scale) hydrogenated graphene, with mobility less than $10 \mathrm{~cm}^{2} /$ V.s and estimated mean free path far beyond the Ioffe-Regel limit [31], suggests some unprecedented robustness of the QHE in damaged graphene [32]. Strong disorder has been indeed considered to be detrimental to the Hall quantization. Recent theoretical papers hint towards a robust QHE even when strong impurities are at a distance smaller than the magnetic length from each other. Moreover, depending on the symmetry class and impurity strength, a splitting of the critical energy within a single Landau level may be expected when disorder introduces valley mixing [33-35], similar to splittings already predicted for certain type of smooth potential [36]. In (quasi-)periodic systems, impurity-engineered Landau levels have been proposed to exist as well [37]. Up to now, the stability and proper quantitative description of such behavior for a completely random distribution of disorder and realistic types of disorder has been lacking, mostly due to computational limitations. 
In this Letter, by using efficient computational methods, we provide a novel insight into the rich physics of QHE in disordered graphene, bringing a comprehensive picture to understand the recent experimental results that have reported unidentified states and QHE features. For single vacancies (SV) and double vacancies (DV) new critical states are observed which are located among and couple over the impurity network, thus precluding the formation of the conventional LLs in pristine graphene (with energies $E_{n}$ ). Instead, the energies of these socalled impurity states lie in between LLs of pristine samples. Additionally, by tuning the magnetic field and the impurity concentration appropriately, a zero energy Hall plateau can be induced. The criticality of the states is studied in the framework of percolation theory [33, 38]. For very weak concentration of DV $(0.05 \%$, conventional pristine extended states may still develop, while for all oher considered DV and SV concentrations, these states become localized, losing the clean graphene quantization.

Model and Methods.- A single-orbital tight-binding (TB) model restricted to $p_{z}$ orbitals is used to describe graphene. SV and DV are modeled in the most simple way by removing the corresponding electron and lattice sites (see insets of Fig. 1). Such simple models allows one to focus on the new impurity states only (avoiding electron-hole asymmetry at higher energy for more realistic models considered separately [39]). Simultaneously, this strategy simplifies the calculation of the off-diagonal conductivity in a newly developed Kubo formalism [40], computed by:

$$
\begin{aligned}
& \sigma_{\mathrm{xy}}=-\frac{2}{V} \int_{0}^{\infty} d t e^{-\eta t / \hbar} \int_{-\infty \infty} d E^{\prime} f\left(E^{\prime}-E\right) \\
& \Re e\left[\left\langle\phi_{\mathrm{RP}}\left|\delta\left(E^{\prime}-\hat{H}\right) \hat{j}_{y} \frac{1}{E^{\prime}-\hat{H}+i \eta} \hat{j}_{x}(t)\right| \phi_{\mathrm{RP}}\right\rangle\right]
\end{aligned}
$$

with the current operator $\hat{j}_{x}=\frac{i e_{0}}{\hbar}[H, \hat{X}](\hat{X}$ the position operator) and $\eta \rightarrow 0$ a small parameter required for numerical convergence. The order- $\mathrm{N}$ method to obtain the longitudinal conductivity is already well established [41, 42]. The effect of the magnetic field is modeled through a Peierls phase substitution [43].

Density of States.- The DOS is estimated for low to high impurity concentrations, for both SV and DV (summarized in Fig. 1). Both impurity models are short-range scatterers and satisfy the valley-mixing condition [3335], but differ in their characteristic features. SV locally

Longitudinal and transverse conductivity.- By combining both the $\sigma_{\mathrm{xx}}$ (dark color) and $\sigma_{\mathrm{xy}}$ (light color) fingerprints in Fig. 2, the energy dependence of localized and extended states is fully clarified. Relevant magnetic field (80T) and impurity concentrations are considered for SV break the sublattice symmetry, induce stronger localization effects, and mimick covalent (or chemical) type of bonding, while DV locally preserve the sublattice symmetry, and better mimick physisorped impurities (through partial ionic bonding). The chosen impurity concentrations all lead to an average distance $d$ between impurities of the order of the magnetic length $\left(l_{B}\right)$ (except for the $0.05 \%$ case of DV), to allow for sufficient interaction between impurities. For the weakest considered impurity concentrations in Fig. 1 (0.05\% for DV and $0.125 \%$ for $\mathrm{SV})$, states still exist at conventional quantization energies $E_{n}$, while these states gradually disappear for higher concentrations. Increasing the concentration, impurity states form at the left and right of each pristine LLs for the DV case, while they only form at the right side for the $\mathrm{SV}$ case (in agreement with the predictions from a (quasi)periodic model [37]). The robustness of the zero energy states in the SV case is explained by the rank-nullity theorem which predicts zero-energy modes (ZEM) to exist for dilute concentrations of SV, while they should not appear for DV [44]. The theorem remains valid under magnetic field because the Peierls phase substitution induces hopping between different sublattices only [45]. To study the criticallity of all these states, time-dependent calculations are necessary.

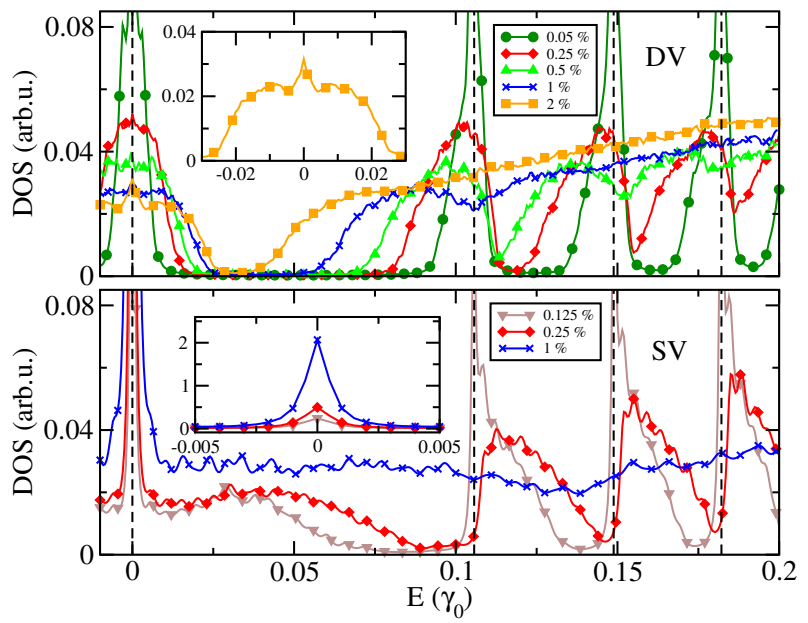

FIG. 1. (color online) DOS curves for SV (lower panel) and DV (upper panel). The curves are symmetric around the CNP, so only the electron side is plotted. Dashed vertical black lines give the energy position $E_{n}$ of LLs in the conventional pristine quantization.

$(0.25 \%)$ and DV (1\%). Rescaled DOS are superimposed for comparison (dashed lines). The new impurity states described by the DOS in Fig. 1 are shown to be extended at their center, leading to plateau steps in $\sigma_{\mathrm{xy}}$ at the energies where they occur. An important observation for 

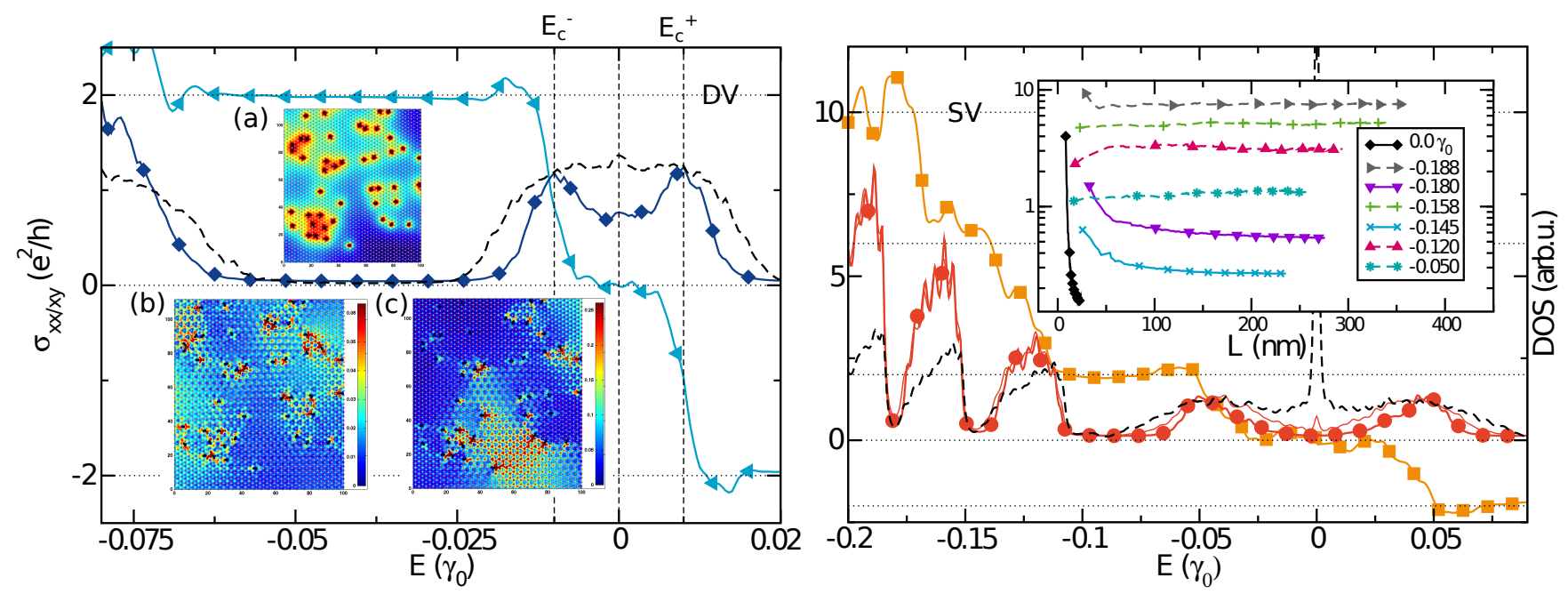

FIG. 2. (color online) $1 \%$ of DV (left panel) and $0.25 \%$ of SV (right panel) at $80 \mathrm{~T}$ : DOS (dashed black lines), $\sigma_{\mathrm{xx}}($ dark color) and $\sigma_{\mathrm{xy}}$ (light color) curves. Horizontal dotted lines give the expected Hall plateaus for IQHE filling facors. Vertical dashed lines in the left panel locate the energies to which panels (b) and (c) correspond. (b) gives the PDOS for $E_{c}^{+}$and $E_{c}^{-}$, while (c) corresponds to $E_{0}$. Inset (a) gives the cumulative distance to impurities as defined in the text. In the right panel, the inset contains the length dependent conductivities $\sigma(L)$ at selected energies. Solid lines correspond to localized energies, while dashed lines correspond to extended states.

both SV (right panel) and DV (left panel) comes from the quantization of this $\sigma_{\mathrm{xy}}$. The impurity states on the left and right of $E_{0}$ only contribute to $2 e^{2} / h$ each to the transverse conductance, indicating they developed from the original LL0, lifting the valley degeneracy (our calculations being spin independent). The critical value of $\sigma_{\mathrm{xx}} \simeq 1.2 e^{2} / h$ for both critical states $\left(E_{c}^{+}\right.$and $\left.E_{c}^{-}\right)$agrees with theory predicting two energies of extended states within the same Landau level when intervalley mixing is present [35]. The other extended impurity states at higher energy contribute by integer multiples of $4 e^{2} / h$ to the transverse conductivity (see $\sigma_{\mathrm{xy}}$ quantization in right panel for SV, similar behavior up to $0.5 \%$ for DV, not shown here). For DV, the detailed behavior at low energy is peculiar in the sense that, even if the DOS does not resolve the double peak structure, the conductivity clearly shows that the states at $E_{c}^{+}$and $E_{c}^{-}$are much more extended than the set of states at $E_{0}$. This unequivocally leads to the formation of plateaus with different slopes in $\sigma_{\mathrm{xy}}[46]$. For the selected case, the slope tends to zero, thus providing only limited contribution to the longitudinal conductivity (around $0.7 e^{2} / h$ for calculated time). To understand the origin of these different behaviors, insets (b) and (c) show the Projected DOS (PDOS) at $E_{c}^{+/-}$ and $E_{0}$ respectively. Inset (a) displays the local impurity density defined as $W_{i}=\sum_{i j}^{N_{\text {imp }}} 1 / d_{i j}$. Blue regions are less affected by impurities than the red ones. A clear correlation is observed between the location of the extended states at $E_{c}^{+/-}$(b) and the impurity density (a), i.e. the extended states mainly spread over the impurity regions of the sample, while the localized states (c) are bound to impurity-free areas. The delocalized nature of states at $E_{c}^{+/-}$(b) is further confirmed by the strongly reduced maximum in the PDOS (0.06 arb.u.) compared to $E_{0}$ (c) where PDOS reaches 0.25 arb.u. To study the extendedness of the SV case, the length dependent conductivities $\sigma(L)$ are plotted in the inset of the right panel. A decreasing behavior indicates localization. Extended state energies in $\sigma(L)$ are confirmed by the plateau transitions in $\sigma_{\mathrm{xy}}$. Albeit having a strong contribution in the DOS at $E_{0}$ for SV, those states turn out to be extremely localized, in sharp contrast with the DV case where the conductivity at $E=0$ remains finite for long times. This different behavior is further scrutinized by comparing the impurity and magnetic field dependence of both systems.

Impurity concentration and magnetic field dependence.- In the very dilute limit (around 0.05\%) impurity states are not close enough to each other to interact significantly, keeping a robust conventional IQHE. Noticable extended impurity states start to appear when $l_{B}$ is of the order of $d$ (as was the case, for $1 \%$, in insets (b) and (c) of Fig. 2 and in panels (a) and (b) of Fig. 3). A similar transition in the dilute regime is expected by decreasing the magnetic field. However, once in the coupled regime, the magnetic field dependence becomes opposite [37, 47] (the $0.5 \%$ at $320 \mathrm{~T}$ is plotted in panel (a) to exemplify this). To study the true percolation of states rooting in the interaction between impurities, the extracted localization lengths (exponential fits) [48] are plotted in Fig. 3(c) for an impurity concentration of $0.5 \%$ (symbols). Percolation theory on graphene predicts a critical exponent $\nu=2.34$ in $\left|E-E_{c}\right|^{-\nu}$ (solid line), which corresponds visually to the numerical behavior for the right tail around $E_{c}^{+}$, exhibiting conventional localization be- 


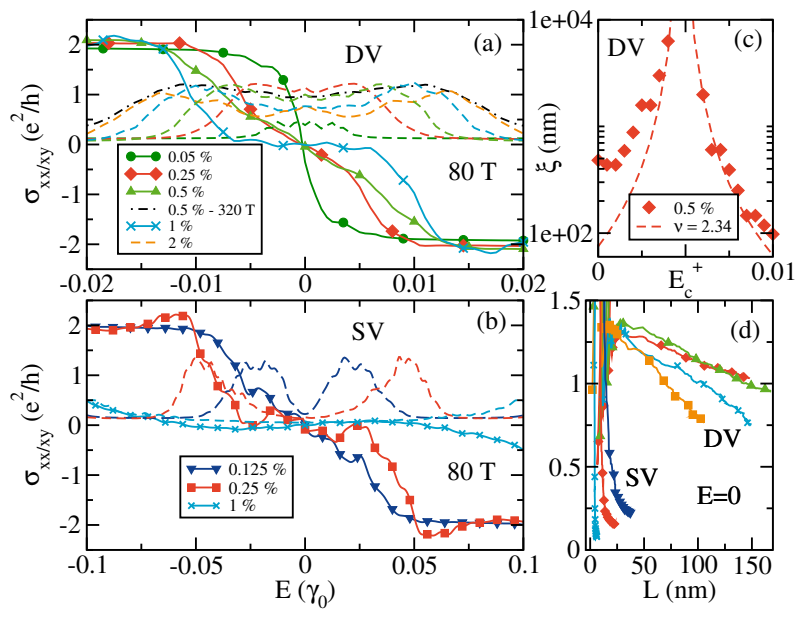

FIG. 3. (color online) $\sigma_{\mathrm{xx}}$ and $\sigma_{\mathrm{xy}}$ for DV case (a) and SV case (b). Estimated localization lengths for $0.5 \%$ at $80 \mathrm{~T}$ with theoretical critical exponantial $(\nu=2.34)$ decay around $E_{c}^{+}$ (c). $\sigma(L)$ for $E_{0}$ for both SV and DV (d)

havior. However, for the left tail, towards $E_{0}$, the agreement is broken for this DV case. This is related to the set of remnant states in the low density regions that behave in a particular manner. The lower right panel compares the localization behavior for SV and DV at this energy. As already mentioned, states are strongly localized for SV on the one hand. This behavior is in agreement with the localization behavior of ZEM predicted at zero magnetic field [49], following a power-law behavior $\sigma \sim L^{\alpha}$ with fitted values of $\alpha$ tending towards -2 . On the other hand, they decay linearly for the DV case, explaining the finite conductivity contributions observed in the left panel of Fig. 2 and panel (a) of Fig. 3. Actually, the highest density concentration $(2 \%)$, increasing the energy split between $E_{c}^{+}$and $E_{c}^{-}$, even allows to resolve the three sets of states (pristine and two impurity sets, as can also be resolved in the DOS in the inset of Fig. 1). This is counterintuitive in the sense that increasing disorder decreases the amount of clean patches in the sample. $\sigma_{\mathrm{xx}}$ at $E_{0}$ remains nevertheless surprisingly robust up to long times, possibly due to the weaker disorder strength for DV, allowing for a remnant connectivity between patches contributing to this finite conductivity.

\section{Conclusion.-}

Acknowledgements.- We acknowledge PRACE for awarding us access to the Curie supercomputing center based in France. The support of Mikolaj Szydlarski from MDLS HPC Dev-Team, France to the technical work is gratefully acknowledged.

* nicolas.leconte@icn.cat

[1] K. v. Klitzing, G. Dorda, and M. Pepper, Physical Re- view Letters 45, 494 (1980).

[2] H. Aoki, Reports on Progress in Physics 50, 655 (1987).

[3] D. J. Thouless, Journal of Physics C 14, 3475 (1981).

[4] B. I. Halperin, Physical Review B 25, 2185 (1982).

[5] R. Prange, Physical Review B 26, 991 (1982).

[6] J. T. Chalker, Surface Science 142, 182 (1984).

[7] R. Joynt and R. Prange, Physical Review B 29, 3303 (1984).

[8] S. V. Iordansky, Solid State Communications 43, 1 (1982).

[9] M. Floser, B. A. Piot, C. L. Campbell, D. K. Maude, M. Henini, R. Airey, Z. R. Wasilewski, S. Florens, and T. Champel, New Journal of Physics 15, 083027 (2013).

[10] R. E. Prange and R. Joynt, Physical Review B 25, 2943 (1982).

[11] K. R. F. and S. Luryi, Physical Review B 25, 7626 (1982).

[12] S. Luryi and K. R. F., Physical Review B 27, 1386 (1983).

[13] S. A. Trugman, Physical Review B 27, 7539 (1983).

[14] G. F. Giuliani, J. J. Quinn, and S. C. Ying, Physical Review B 28, 2969 (1983).

[15] M. Tsukada, Journal of the Physical Society Japan 41, 1466 (1976).

[16] K. Novoselov, A. Geim, S. Morozov, D. Jiang, M. Katsnelson, I. Grigorieva, S. Dubonos, and A. Firsov, Nature (London), 197 (2005).

[17] Y. Zhang, Y.-W. Tan, H. Stormer, and P. Kim, Nature 438, 201 (2005).

[18] M. Goerbig, Review of Modern Physics 83, 1193 (2011).

[19] J. W. McClure, Physical Review 104, 666 (1956).

[20] A. H. Castro Neto, N. M. R. Peres, K. S. Novoselov, and A. K. Geim, Review of Modern Physics 81, 109 (2009).

[21] P. Rickhaus, M. Weiss, L. Marot, and C. Schönenberger, Nano Letters 12, 1942 (2012).

[22] Y. Barlas, K. Yang, and A. H. MacDonald, Nanotechnology 23, 052001 (2012).

[23] Y. Zhang, Z. Jiang, J. P. Small, M. S. Purewal, Y.W. Tan, M. Fazlollahi, J. D. Chudow, J. A. Jaszczak, H. L. Stormer, and P. Kim, Physical Review Letters 96, 136806 (2006).

[24] K. Nomura and A. H. MacDonald, Physical Review Letters 96, 256602 (2006).

[25] A. F. Young, C. R. Dean, L. Wang, H. Ren, P. CaddenZimansky, K. Watanabe, T. Taniguchi, J. Hone, K. L. Shepard, and P. Kim, Nature Physics 8, 550 (2012).

[26] J. Jung and A. H. MacDonald, Physical Review B 80, 235417 (2009).

[27] S. Maro, arXiv:1410.4415 (2014).

[28] G. Li and E. Y. Andrei, Nat. Phys. 3, 623 (2007).

[29] Y. Nam, J. Sun, N. Lindvall, S. Jae Yang, D. Kireev, C. Rae Park, Y. Woo Park, and A. Yurgens, Applied Physics Letters 103, 233110 (2013).

[30] S. Jung, G. M. Rutter, N. N. Klimov, D. B. Newell, I. Calizo, A. R. Hight-Walker, N. B. Zhitenev, and J. A. Stroscio, Nat. Phys. 7, 245 (2011).

[31] A. Ioffe and A. Regel, Progress in Semiconductors 4, 237 (1960).

[32] J. Guillemette, S. S. Sabri, B. Wu, K. Bennaceur, P. E. Gaskell, M. Savard, P. L. Lévesque, F. Mahvash, A. Guermoune, M. Siaj, R. Martel, T. Szkopek, and G. Gervais, Physical Review Letters 110, 176801 (2013).

[33] M. Koshino and T. Ando, Phys. Rev. B 75, 033412 (2007).

[34] P. Goswami, X. Jia, and S. Chakravarty, Phys. Rev. B 76, 205408 (2007). 
[35] P. M. Ostrovsky, I. V. Gornyi, and A. D. Mirlin, Physical Review B 77, 195430 (2008).

[36] D. K. K. Lee, Phys. Rev. B 50, 7743 (1994).

[37] A. L. C. Pereira and P. A. Schulz, Physical Review B 78, 125402 (2008).

[38] J. T. Chalker and P. D. Coddington, Journal of Physics C: Solid State Physics 21, 2665 (1988).

[39] N. Leconte, F. Ortmann, A. Cresti, J.-C. Charlier, and S. Roche, 2D Materials 1, 021001 (2014).

[40] F. Ortmann and S. Roche, Physical Review Letters 110, 086602 (2013).

[41] S. Roche, Physical Review B 59, 2284 (1999).

[42] Z. Fan, A. Uppstu, T. Siro, and A. Harju, Comp. Phys. Comm. 185, 28 (2014).
[43] J. M. Luttinger, Physical Review B 54, 814 (1951).

[44] G. Trambly De Laissardiére and D. Mayou, Mod. Phys. Lett. B 25, 1019 (2011).

[45] V. Pereira, J. Lopes Dos Santos, and A. Castro Neto, Physical Review B 77, 115109 (2008).

[46] X. Jia, P. Goswami, and S. Chakravarty, Phys. Rev. Lett. 101, 036805 (2008).

[47] D. A. Bahamon, A. L. C. Pereira, and P. A. Schulz, Physical Review B 79, 125414 (2009).

[48] N. Leconte, A. Lherbier, F. Varchon, P. Ordejon, S. Roche, and J.-C. Charlier, Physical Review B 84, 235420 (2011).

[49] A. Cresti, F. Ortmann, T. Louvet, D. Van Tuan, and S. Roche, Phys. Rev. Lett. 110, 196601 (2013). 